\title{
Fundamental Limits in The Rendering of Virtual Haptic Textures
}

\author{
Gianni Campion and Vincent Hayward \\ Haptics Laboratory \\ Centre for Intelligent Machines, \\ McGill University, Montréal, Canada \\ E-mail: $\{$ champ|hayward\}@cim.mcgill.ca
}

\begin{abstract}
We discuss the properties of force-feedback haptic simulation systems that fundamentally limit the re-creation of periodic gratings, and hence, of any texture. These include sampling rate, device resolution, and structural dynamics. Basic sampling limitations are analyzed in terms of the Nyquist and the Courant conditions. The analysis proposes that noise due to sampling and other sources injected in the system may prevent it to achieve acceptable performance in most operating conditions, unless special precautions such as the use of a reconstruction filter, make the closed-loop more robust to noise. The structural response of a PHANTOM 1.0A device was such that no such filter could be found, and the system introduced heavy distortion in gratings as coarse as $10 \mathrm{~mm}$. The Pantograph Mark-II device having more favorable structural properties could reliably create gratings between 1 and $10 \mathrm{~mm}$.
\end{abstract}

\section{Introduction}

Texture is important in haptic simulations because, like frictional properties or shape, it is a key attribute of real and simulated objects. In computer graphics, much work was, and still is, aimed at texturing images, but in haptics, despite much past research $[16,18,9,6,19,13,17,7]$, the question of realism has only been recently addressed [4].

In this paper, we discuss the characteristics of a system which set absolute limits on what can be rendered with force feedback devices. Because these devices operate on sampled data both in time and in space, artifacts can arise when a user interacts with virtual objects using a mechanical interface which necessarily interposes its own dynamics between the object and the user's fingers.

In general, the factors that limit the synthesis of texture independently from any particular method are: system sampling period, sensor noise (related to resolution), output torque resolution, device structural dynamics, and other factors such as backlash in the joints.
What we found is that for commonly available devices, the finest textures that can be reliably and accurately synthesized without special precautions are surprisingly and perilously coarse.

\section{Basic Sampling}

All texture synthesis algorithms rely on a "generating function" $g(x)$ used to compute a force from a position. It can be periodic, stochastic, or a mixture of both. To analyze sampling effects, we must assume that $g(x)$ is $C^{1}$ given that it must be finitely sampled and then reconstructed during synthesis. Without loss of generality, we also assume $|g(x)| \leq 1, \forall x$, and that it is band-limited. For this reason, it is sufficient to look at the case of a sinusoidal grating. The observations made next extend to any periodic grating and to band-limited stochastic textures since they can be decomposed in a finite sum of sinusoids.

Consider scanning a grating of spatial period $1 / k$ at an unknown velocity $v$. The system is sampled at rate $1 / \mathcal{T}$. This is equivalent to sampling a progressive wave with wave number $k$ at a stationary point, see Figure 1 .

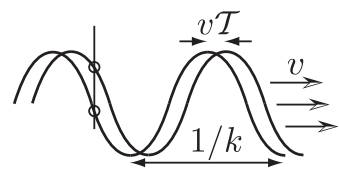

Figure 1: Circles represent places where the grating is ideally sampled.

There exists a critical velocity $v_{T}$ at which the discretized grating vanishes,

$$
v_{T}=\frac{1}{k \mathcal{T}} .
$$

Precise reconstruction is possible only if $v \ll v_{T}$, i.e. $\alpha v<v_{T}$. The Nyquist criterion states that to reconstruct a signal, we need $\alpha \geq 2$. If this condition is not met then 
the generating function cannot be reconstructed. The accuracy of the reconstruction, however, is not guaranteed by the Nyquist criterion. We must pick a safer limit, since the Nyquist rate can be approached only when using nearideal reconstruction filters. Since, typically, the electromechanical transfer function of the haptic device serves as a less-than-ideal reconstruction filter, $\alpha \approx 10$ provides a reasonable limit. In this condition,

$$
g(x(t))=\sin (2 \pi k x(t))
$$

can be well approximated by its discrete-time counterpart

$$
g_{i}\left(x_{i}\right)=\sin \left(2 \pi k x_{i}\right),
$$

where the $x_{i}$ are the successive position samples measured at rate $1 / \mathcal{T}$ by the device. When $v \ll v_{T}$, then the temporal frequency is $f=v k \ll 1 / \mathcal{T}$ so the system operates far from the Nyquist rate, that is:

$$
\alpha k v \mathcal{T}<1
$$

Example 1 Simulate a grating with a $1.0 \mathrm{~mm}$ pitch at $1.0 \mathrm{kHz}\left(\mathrm{k}=10^{3}, \mathcal{T}=10^{-3}\right)$. Staying sufficiently far under $v_{T}$ requires the scanning speed to remain under $0.1 \mathrm{~m} / \mathrm{s}$ - a rather low speed by human standards. Simulating a finer pitch of $0.1 \mathrm{~mm}$ (grooves of a vinyl record) would require the speed to remain under $0.01 \mathrm{~m} / \mathrm{s}$.

So far we assumed that the device measured the $x_{i}$ perfectly. In practice, any device has limited resolution. Let's consider that the device makes quantized measurements with a resolution $\delta$, the smallest displacement at the tip that can be reliably detected. By an analogous reasoning, in the absence of a space reconstruction filter, then we would require to have at least $\beta \approx 10$ samples within one spatial period:

$$
\beta k \delta<1
$$

Example 2 A device having a resolution of $10 \mu \mathrm{m}(\delta=$ $10^{-5}$ ) at best can accurately reconstruct a $0.1 \mathrm{~mm}$ grating $\left(k=10^{-4}\right)$, all other sources of error ignored.

Since a haptic simulation system essentially solves a numerical problem discretized in time and space, it is subject to the Courant-Friedrichs-Lewy condition:

$$
\delta>v_{C} \mathcal{T}, \quad v_{C}<\frac{\delta}{\mathcal{T}},
$$

which is equivalent to considering that the velocity cannot be known better than one velocity quantum $\delta / \mathcal{T}$. We can conclude that increasing the sampling rate of the simulation may not improve it, if the resolution of the device is not increased as well, while making it more difficult to estimate velocity $[14,5]$.
Example 3 If we sample at $10.0 \mathrm{kHz}\left(\mathcal{T}=10^{-4}\right)$, for the device to resolve movements at $v=0.1 \mathrm{~m} / \mathrm{s}$, its resolution must be better than $10 \mu \mathrm{m}\left(\delta=10^{-5}\right)$.

Therefore, a trade-off exists between device resolution, sampling rate, scanning speed and grating period. We can reconcile these observations by combining the safe velocity of Eq (4) given by the Nyquist criterion with the critical velocity of Eq (6) given by the Courant condition ( $\alpha=v \mathcal{T} / \delta$ is the Courant number) and find that, indeed, the device should have a resolution such that

$$
\alpha k \delta<1
$$

Output quantization can also cause similar problems. If we call $b$ the smallest step of force that can be resolved, we should impose that there are at least $\gamma$ steps within the rendered force amplitude $A$ :

$$
\gamma b<A
$$

These constraints can easily be extended to non-periodic textures by knowing the spectrum of the generating function.

An estimate of how well the generating function can be reconstructed is found by considering that each measurement is made with an error on $\delta_{i}$ on the true position $x_{i}=x+\delta_{i}$. Assuming that $\delta_{i}$ is small:

$$
\begin{aligned}
g\left(x+\delta_{i}\right) & \approx g(x)+\partial g / \partial x \delta_{i}=g(x)+\epsilon \\
& \approx \sin (2 \pi k x)+2 \pi \delta_{i} k \cos (2 \pi k x) .
\end{aligned}
$$

Thus, the discrete grating has an error term $\epsilon$ :

$$
|\epsilon|_{\infty}=2 \pi k \max _{i}\left|\delta_{i}\right|=2 \pi \delta k .
$$

For a given device resolution the error is amplified if the spatial period is smaller. This error is dominated by space quantization when $v$ is small, $\epsilon \simeq 2 \pi / \beta$ and by time quantization when $v$ is large: $\epsilon \simeq 2 \pi / \alpha$ (Eqns (5) and (7)).

Example 4 Simulate a $1.0 \mathrm{~mm}$ grating with a device with a resolution of $10 \mu \mathrm{m}\left(\delta=10^{-5}, k=10^{3}, \beta=100\right)$. The relative error is 0.06 , that is $6 \%$. If we try to simulate a finer grating of $0.1 \mathrm{~mm}$ pitch $(\beta=10)$, the error become $60 \%$ which is hardly acceptable. Since $\delta$ is random, the simulation is noisy.

\section{Feedback Dynamics}

While many results were found in the past by considering a device to be a damped mass (a rigid body plus some dissipation), when it comes to simulating textures, it is clear that device behavior at high frequencies matters, and that the rigid body assumption may no longer hold. 
A texture simulation system operates in closed loop, hence, feedback control theory can be useful to analyze its properties. Consider the classical set-up as in Figure 2 $[8,1]$. There, $r$ represents an input to be tracked, $y$ the output, $d$ the noise injected in the system normalized at the output, $e$ the error, and often one adds an external disturbance $n$ to the nominal command $u$ giving $v$. The loop is closed around a controller $C$ and a plant $P$.

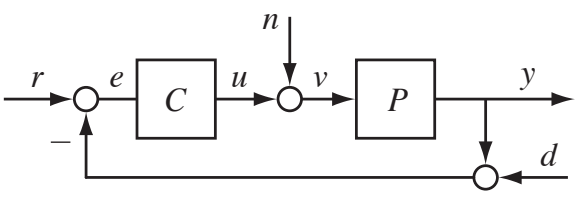

Figure 2: System with feedback.

In texture simulation, sources for $d$ (encoder noise) and $n$ (numerical noise and other disturbances such as friction or analog-to-digital reconstruction noise) have been identified in the preceding section. Two of these transfer functions are of particular importance, that of $d$ to $y$ (transmission function $T$ ) and that of $d$ to $v$. Calling $L=P C$,

$$
T \triangleq-\frac{y}{d}=\frac{L}{1+L}, \quad \frac{v}{d}=\frac{T}{P}=\frac{C}{1+L} .
$$

Similar manipulations would allow us to evaluate the effect of $n$ on the closed-loop system.

We now relate the general diagram to the case of a haptic texture simulation system as in Figure 3. In most instances, actuators and sensors are co-located, so $P$ represents the device transfer function from motor current command to motor movement (including the amplifiers).

While the closed-loop function $T$ is crucial, what ultimately matters is what the user feels, thus the system response should be considered from the device tip (measured with an accelerometer [10]). This corresponds to an openloop transfer function $R$ which is related to the displacement $h$ of skin via a double integrator.

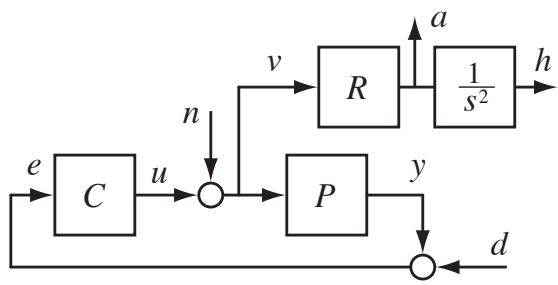

Figure 3: Haptic simulation set-up.

The functions $R$ and $P$ co-vary as a function of many factors: as a function of the configuration, of the load, and in particular, from the mere act of touching the device. This is because devices naturally have structural characteristics with high-Q resonances (e.g. [12, 3]), possibly also arising from the motors [2]. Structural dynamics are uncertain and resonances can shift unpredictably. A systematic design for a filter $H$ would be difficult ( $\mu$-synthesis, convex optimization, or other methods), and if at all possible, will have to be conservative. The structural dynamics of a device, both amplify noise in the close-loop, and distort the signal in open loop. Since it is hard to robustly compensate for structural dynamics beyond the first mode, this introduce another fundamental limit. Calling $F_{0}$, the frequency of the first mode of the device:

$$
v k<F_{0}
$$

The generating function yields a force signal of maximum amplitude $A$, the intensity of the resulting grating. Linearizing $g$ around a particular true position $x$ as in Eq. (10) (small signal analysis), gives us a block $C(r=0)$ corresponding to the slope of the texture generating function times the intensity factor $A$ (the Jacobian of $g$ in the case of multidimensional texture simulation) plus a reconstruction filter $H$ that is typically ignored (i.e. $H=1$ ):

$$
C=A \frac{\partial g}{\partial x} H
$$

The finer the texture, the higher the instantaneous loop gain, which varies with $k$ for the simple grating (Eq. (10)). This introduces a new constraint that says that in order to keep the loop gain independent from any particular grating, then $A$ must be reduced proportionally to $k$. Calling $A_{0}$ the maximum acceptable stiffness (e.g. for stability):

$$
A k<A_{0}
$$

\section{Experiments}

We applied the foregoing analysis using two haptic devices: the PHANTOM ${ }^{\circledR}$ from Sensable and the recently rebuilt Pantograph Mk-II [2], see Figure 4. The PHANTOM (model 1.0A) is a haptic device designed to explore 3D objects which is frequently used in research laboratories. It has cable drives that provide torque amplification and is statically balanced [15]. The Pantograph is a direct-driven planar device designed to render surfaces [11].

\subsection{Device Characterization}

Sampling rate. The devices were both interfaced to a personal computer ( $2.5 \mathrm{GHz}$ P-IV processor), via a PCI proprietary interface for the PHANTOM, and via a "hardwarein-the-loop" PCI card from Quanser Inc. (Model Q8) for the Pantograph. The system was running RTLinux 3.2pre3 that enabled hard real-time sampling rates up to $100 \mathrm{kHz}$. In all cases, however, the control loops ran at $10 \mathrm{kHz}$. We found that at $10 \mathrm{kHz},\left(\mathcal{T}=10^{-4}\right)$ RTLinux ran the hardrealtime thread with a period jitter never exceeding $0.5 \%$. 


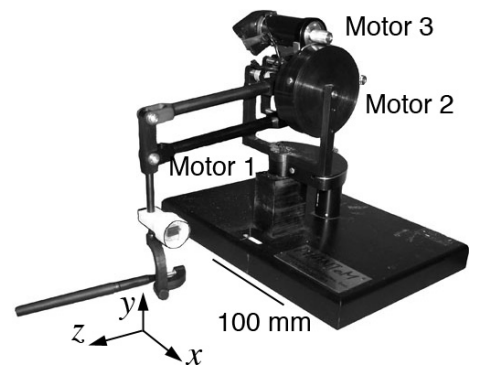

Sensable PHANTOM 1.0A

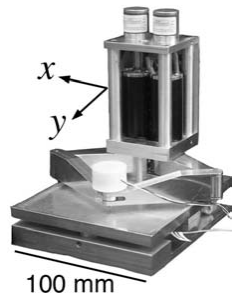

Pantograph Mk-II
Figure 4: Devices used for the experiment

Resolution. Given $\Delta$ the vector of $p$ individual joint resolutions and $\mathbf{J}(q)$ the device Jacobian, the resolution was estimated using:

$$
|\delta|_{\infty}=\max _{\mathbf{l} \in\{-1,1\}^{p}}(|| \mathbf{J}(q) \operatorname{diag}(\Delta) \mathbf{l}||) .
$$

For the PHANTOM 1.0A (4,000 CPR encoders and accounting for joint ratios), the nominal resolution was found to vary between 40 and $70 \mu \mathrm{m}$, Figure 5. For the Pantograph Mk-II with $2^{16}$ CPR encoders, the nominal resolution was found to vary between 9 and $13 \mu \mathrm{m}$, see Figure 5. Note that, in essence, these figures express resolutions which are guaranteed not to be achieved in practice.
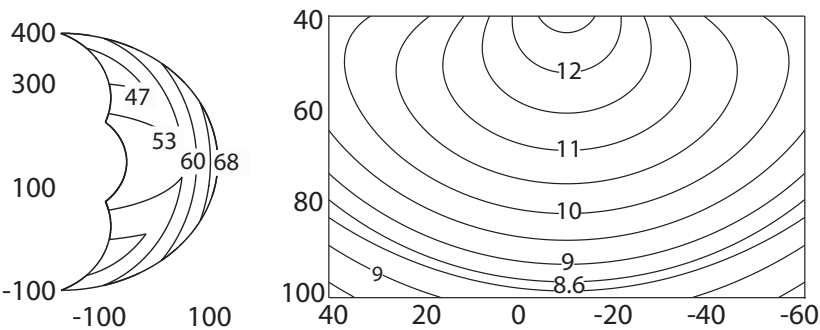

Figure 5: The nominal resolution in $\mu \mathrm{m}$ of the PHANTOM in the mid-sagittal plane (left) and of the Pantograph (right) plotted over their workspaces indicated in millimeters.

Nevertheless, the safety factor $\beta$ found in Section 2 is greater than 10 for both devices for textures whose smallest spatial period is $1 \mathrm{~mm}$. Given 12 bit analog-to-digital converters, the PHANTOM's force granularity varied between 13 and $20 \mathrm{mN}$ for the Pantograph's between 2 and $5 \mathrm{mN}$.

Structural Response. The devices were tested using chirp excitation (DSP Technology Inc. system analyzer, SigLab Model 20-22). An accelerometer (Analog Device; model ADXL250) was clamped to the distal end of the PHANTOM using a light-weight fixture to minimize effects on the response. For the Pantograph, the same accelerometer was embedded in the finger interface plate. This enabled us to measure directly the open-loop transfer func- tion $R$. We did not attempt to measure $P$ since encoders do not have enough resolution in the high frequencies where displacements are vanishingly small.

For the PHANTOM, the condition where the device was lightly loaded by a rubber-band (slightly taught to keep it in place) is reported for all directions. It is also shown for the $z$ direction when it was loaded by a grip.

The results, Figure 6, indicate that the lowest structural anti-resonance was around $30 \mathrm{~Hz}$ in the $z$ direction and that there was a resonance at $100 \mathrm{~Hz}$ on the $x$ axis. Naturally, there were many others modes extending up to $700 \mathrm{~Hz}$, changing in frequency, magnitude, and Q, according to the loading conditions.
Free response in $x$

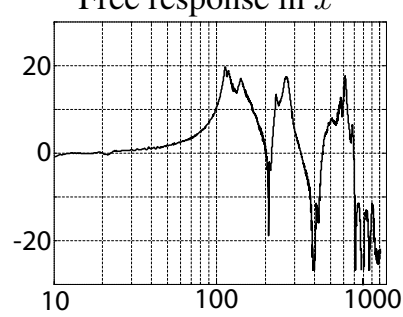

Free response in $z$

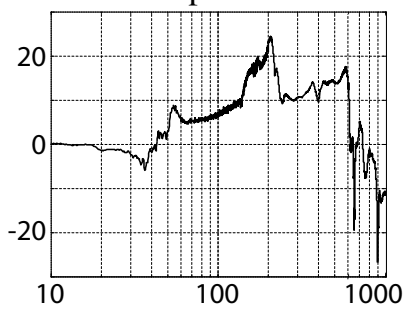

Free response in $y$

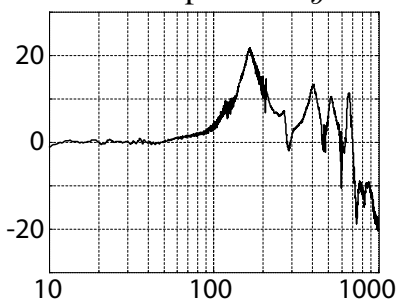

Loaded response in $z$

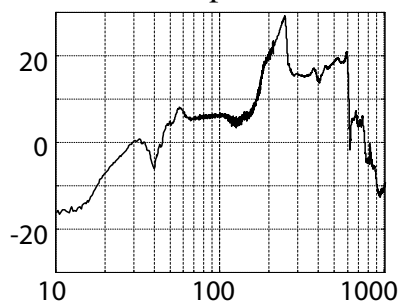

Figure 6: Response examples of the PHANTOM.

For the Pantograph, the first condition also was when the plate was held with by a rubber-band, the second was when a finger touched the plate lightly, and the third when the finger pressed hard, see Figure 7. There were two dominant resonances, one around $400 \mathrm{~Hz}$ (possibly introduced by the motors) and one around $900 \mathrm{~Hz}$. The second resonance is puzzling because it magnified instead of being damped out when pushing harder on the plate.

\subsection{Effect of a reconstruction filter}

For the PHANTOM, candidate filters $H$ that could provide a reasonable open loop response while making the closed loop more robust given the structural imperfections at $30 \mathrm{~Hz}$ and $100 \mathrm{~Hz}$ were found to introduce too much phase delay, making the system unstable.

For the Pantograph, because the response was well behaved until $400 \mathrm{~Hz}$, a filter could be empirically designed (Butterworth order 10, $400 \mathrm{~Hz}$ cut-off, ran at $10 \mathrm{kHz}$ ). With this filter, the Pantograph could in principle render a $1 \mathrm{~mm}$ grating with an error of $8 \%$ and a speed of $0.4 \mathrm{~m} / \mathrm{s}$. 


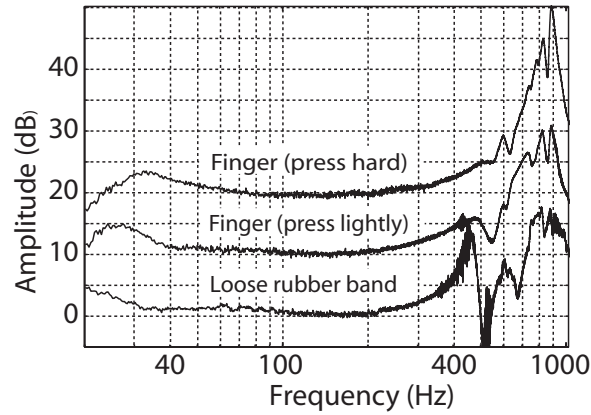

Figure 7: Dynamic response of the Pantograph. The three responses are shown offset by $10 \mathrm{~dB}$ for clarity.

Figure 8 shows the effect of adding the filter when exploring a $1 \mathrm{~mm}$ sinusoidal grating. Without it, the rendered texture is essentially uncorrelated with the $g(x)$. The left panels show that the rendered acceleration is essentially determined by the noise injected in the system. It is magnified and almost exclusively concentrated in the $900 \mathrm{~Hz}$ band, as shown by the acceleration spectrum. With the filter, the rendered texture, see the right panels, has the expected shape and most of its energy is in the correct frequency band.
Acceleration (No filter)

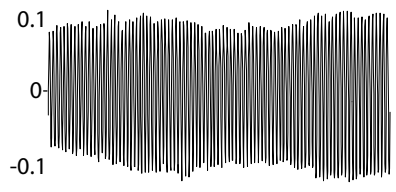

0

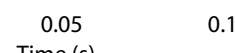

Time (s)

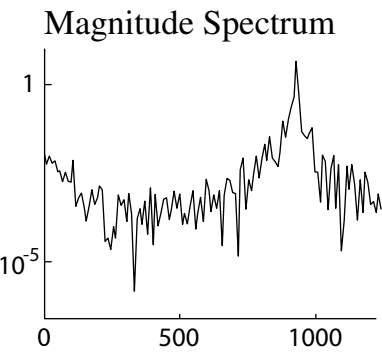

Acceleration (With filter)

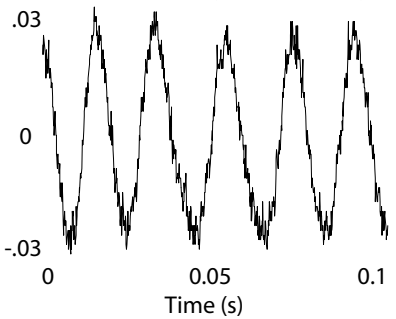

Magnitude Spectrum

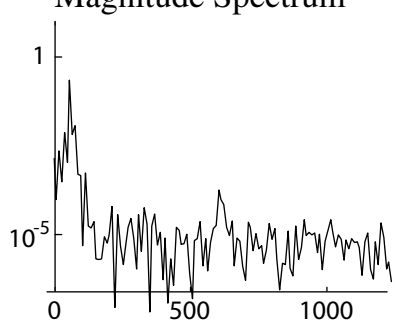

Figure 8: Pantograph: Effect of $400 \mathrm{~Hz}$ Butterworth filter: Left without filter, right with the filter in the loop.

\subsection{Comparative tests}

We discuss here a sample of results for the two devices, for two grating periods $(1 \mathrm{~mm}$ and $10 \mathrm{~mm})$, and for two different scanning speed ranges (from $0.06 \mathrm{~m} / \mathrm{s}$ to $0.9 \mathrm{~m} / \mathrm{s}$ ), yielding eight cases. In Figure 9 each force waveform that was to be rendered is shown next to the corresponding acceleration waveform. Under each panel also is the corresponding spectrum.

We rendered $f(s)=A \sin (2 \pi k s(t))$, a onedimentional grating. For the Pantograph the force was always in the $x$ direction and the $s(t)$ was simply $x(t)$. For the PHANTOM, the force was always radial from the first joint and horizontal, and $s(t)$ was the distance from the first joint axis. The grating was rendered with just motors 2 and 3 (the force being always in the 4-bar plane) and depended mostly on the $z$ axis dynamics because we kept the position close to the neutral point. The values for $A$ where $0.9 \mathrm{~N}$ for the PHANTOM and $0.4 \mathrm{~N}$ for the Pantograph, providing a conservative $\gamma$ margin. These values also provided similar tactile intensities as well as similar stability margins with the two devices.

In the following figures, the upper-left panels always show the force command that corresponds to signal $u$ in Figure 3. The upper-right panel shows to corresponding measured acceleration, signal $a=s^{2} h$ in Figure 3. In the upper panels, the second trace plotted in dashed line shows acceleration in an orthogonal direction.

Coarse grating - slow speed. The examples corresponded to slowly scanning a $10 \mathrm{~mm}$ grating which, in principle, could be thought of providing the best case possible. For the PHANTOM, while the force command was not sinusoidal, as one should expect when moving slowly through an oscillatory force field (in this frequency range, the elasticity of the finger is significant), the resulting acceleration bore little resemblance with the expected signal. We see that the PHANTOM introduced high frequency noise not present in the commanded signal. The Pantograph showed the same overall behavior, but the shape of the acceleration signal is much better.

Coarse grating - fast speed. These examples used the same grating, but this time, the experimenter attempted to move at a relatively fast speed. As expected, the force command signal was then confined to a narrow band because the movement 'punches through' the grating, and this was the case for both devices. For the PHANTOM, the rendering was acceptable in the direction of the movement as shown by the magnitude spectrum of the acceleration. There was a noticeable defect in the orthogonal direction where, somehow, significant energy spills over in the $700 \mathrm{~Hz}$ range. The observed spectrum spread is the hallmark of a nonlinear system. The cross-talk in orthogonal directions also changes the signal frequency. For the Pantograph, the rendering was nearly perfect.

Fine grating - slow speed. These examples corresponded to the case of a $1 \mathrm{~mm}$ grating scanned at slow speed. The force to be rendered by the PHANTOM suffers from quantization noise (even if $\beta>10$ ) and some instability in the $300 \mathrm{~Hz}$ range. This yielded an acceleration signal which was essentially unrelated to the desired 
result, since most of the signal energy was in the $600 \mathrm{~Hz}$ band where in fact it should have been in the $100 \mathrm{~Hz}$ band. For the Pantograph, the grating was faithfully reproduced.

Fine grating - fast speed. These examples used the same $1 \mathrm{~mm}$ grating but scanned at fast speed. Going against what intuition would have suggested, for the PHANTOM, the commanded force was almost noise-free with the exception of some harmonic distortion in the $700 \mathrm{~Hz}$ band. The rendered acceleration was also almost distortion-free in the scanning direction but there was significant cross-talk in an orthogonal direction. This can be explained by the fact that for this particular grating and scanning speed, the device operated in a band which was free of structural modes for that direction but excited modes in another. The Pantograph rendered the grating faithfully.

\subsection{Discussion}

These examples, among many others that cannot be discussed here but which reveal a number of other effects, clearly indicate that the rigid body assumption is not acceptable when rendering textures with medium-scale force feedback systems. If the bandwidth inside which the system can be considered a rigid body is sufficiently large, then we can cut-off the response so that Condition (13) holds, then as long as Conditions (5) and (7) hold, the rendered texture will be accurate. Under any other circumstances we will run the risk of rendering a signal which is quite different from what was programmed in $g(x)$. In that, we concur with the opinion expressed in [4], that human studies about the perception of textures using systems of designs and scales comparable to that of the PHANTOM may have been tainted. By comparing the force signal to the acceleration, we have results that are algorithmindependent and relate better to fundamental limits.

\section{Conclusion}

Using the analogy between scanning a texture and a wave traveling at a variable speed, we used the Nyquist and the Courant conditions to derive relationships that state the conditions under which a texture can possibly be rendered. The limits imposed by the sampling theory were found to be insufficient to guarantee the correct rendering of a texture in general. A haptic device is a mechanical system which cannot be approximated by a rigid body when excited by fast signals. The Jacobian of the rendering function essentially determines the gain in the closed loop, therefore the complete system is subject to the constraints of feedback dynamics when significant noise is injected in a system which is structurally non-robust.

What we found can be summarized as follows. Given $k$ the spatial frequency of a grating, $\mathcal{T}$ the system sam- pling period, $v$ the scanning velocity, $\delta$ the device resolution, $b$ the force resolution, $\alpha$ a temporal safety factor (at least 2, most likely 10), $\beta$ a spatial safety factor (at least 2 , most likely 10 or more), $\gamma$ a force reconstruction safety factor (at least 10), $A$ the desired force amplitude the rendered grating, $A_{0}$ the maximum control stiffness, and $F_{0}$ the first mode of the device, then Table 1 summarizes the limits that cannot be exceeded in order to make it possible to render a given grating with a given device. These limits do not guarantee that the grating question will be rendered correctly, but if one of these limits is exceeded it is highly likely that it will not be the case.

Table 1: Summary of limits.

$\begin{array}{lr}\text { Scanning velocity limit ........ } & \alpha k v \mathcal{T}<1 \\ \text { Low speed reconstruction limit } & \beta k \delta<1 \\ \text { High speed reconstruction limit } & \alpha k \delta<1 \\ \text { Force reconstruction limit ...... } & \gamma b<A \\ \text { Gain limit .................. } & A k<A_{0} \\ \text { Device structural limit ........ } & v k<F_{0}\end{array}$

As an example, the PHANTOM which, in principle, has enough resolution in time and space to render correctly textures up to $1 \mathrm{~mm}$ was found to render incorrectly textures as coarse as $10 \mathrm{~mm}$. With another device, the Pantograph, which has a much higher structural bandwidth, it was possible to find a reconstruction filter which robustified the system under all reasonable operating conditions, although finding optimal filters that can take into account both the open loop and the closed loop behavior of a given haptic system remains a daunting task.

This study also suggested a new performance measure for haptic devices, namely the smallest grating that can be rendered reliably. For the PHANTOM, we were not able to determine it. For the Pantograph, this number is around $1 \mathrm{~mm}$, still a far cry indeed from what is needed to simulate a realistic texture imitating surface finishes, such as that of wood, for example.

\section{Acknowledgments}

This research was supported in part by the Institute for Robotics and Intelligent Systems, and the Natural Sciences and Engineering Research Council of Canada. G. Campion is the recipient of a PRECARN Inc. scholarship.

The authors would like to thank Prof. Hong Z. Tan of Purdue University for insightful comments on an early draft of this paper and the reviewers for their excellent suggestions. The authors are indebted to Prof. David Ostry of McGill University for letting us use his laboratory's PHANTOM, to Prof. Keyvan Hashtrudi-Zaad of Queen's University for showing us how to interface it; to Hsin-Yun Yao of the Haptics Lab at McGill for custom-packaging the miniature accelerometers, and to Andrew Havens Gosline also from the Haptics Lab for proof-reading the paper. 
Proc. First Joint Eurohaptics Conference and Symposium on Haptic Interfaces for Virtual Environments and Teleoperator Systems WHC'05, pp. 263-270.

PHANTOM

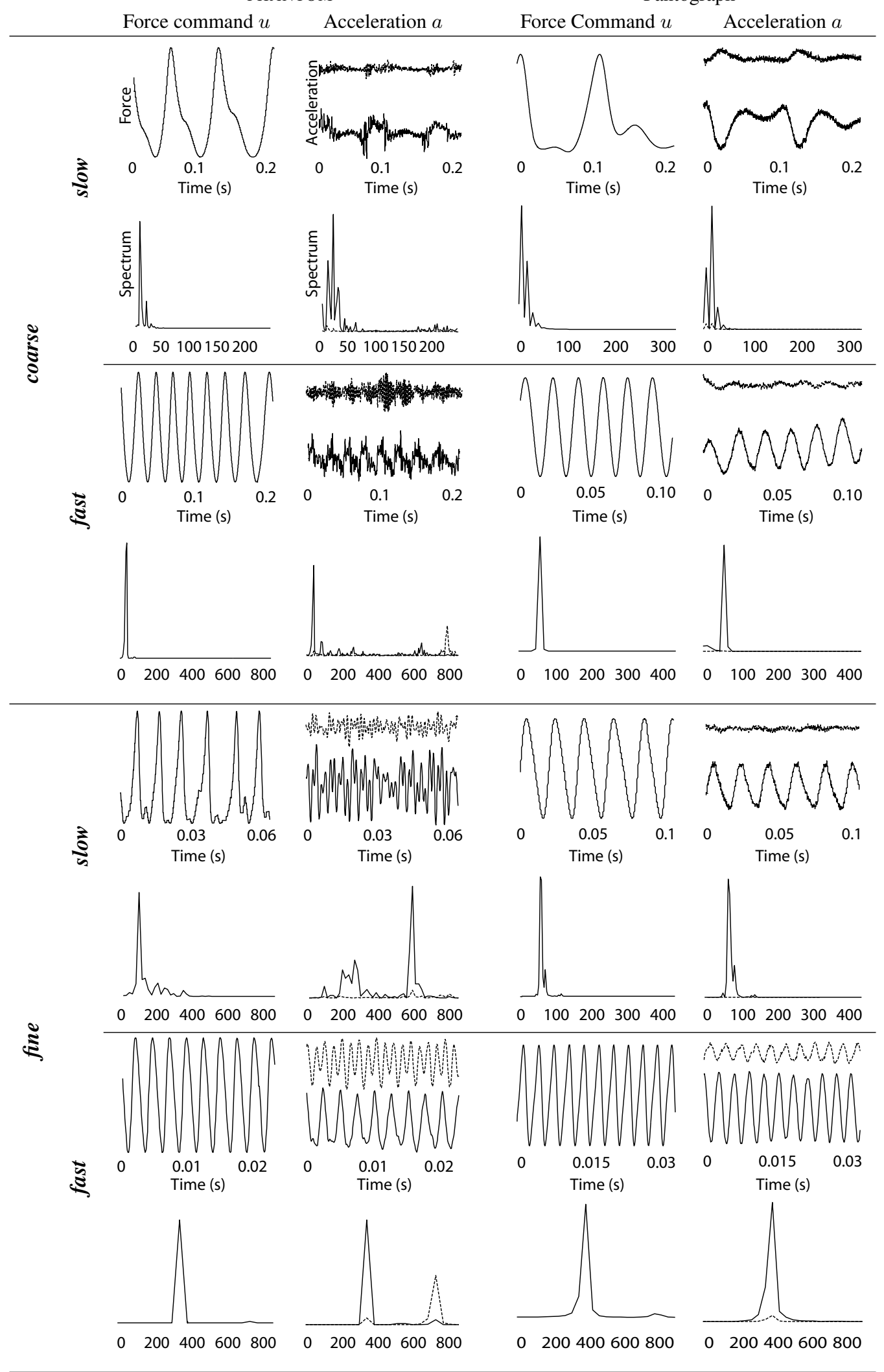

Figure 9: Summary of the eight testing conditions for the two devices. 
The authors would like to acknowledge Seigo Harashima from RICOH Company for many keen discussions on haptic textures.

\section{References}

[1] C. Barratt and S. Boyd. Closed-loop convex formulation of classical and singular value loop shaping. In Leondes C. T., editor, Digital and Numeric Techniques and Their Applications in Control Systems, Part 1, 1993.

[2] G. Campion, Q. Wang, and V. Hayward. The Pantograph Mk-II: A haptic instrument. 2005. In preparation.

[3] M. C. Cavusoglu, D. Feygin, and F. Tendick. A critical study of the mechanical and electrical properties of the PHANToM haptic interface and improvements for high performance control. Presence: Teleoperators and Virtual Environments, 11(6):555-568, 2002.

[4] S. Choi and H. Z. Tan. Perceived instability of virtual haptic texture. i. experimental studies. Presence: Teleoperators \& Virtual Environments, 13(4):395415, 2004.

[5] J.E. Colgate and J.M. Brown. Factors affecting the Z-width of a haptic display. In Proc. IEEE Int. Conf. Robotics and Automation, pages 3205-3210, 1994.

[6] M.A. Costa and M.R. Cutkosky. Roughness perception of haptically displayed fractal surfaces. In Proc. ASME IMECE Symposium on Haptic Interfaces for Virtual Environments and Teleoperator Systems, 2000.

[7] A. Crossan, J. Williamson, and R. Murray-Smith. Haptic granular synthesis: Targeting, visualisation and texturing. In IEEE Computer Society, editor, In Proc. International Symposium on Non-visual \& Multimodal Visualization, pages 527-532, 2004.

[8] S. P. Doyle, B. A. Francis, and A. R. Tannenbaum. Feedback Control Theory. Macmillan Publishing, 1992.

[9] J. P. Fritz and K. E. Barner. Stochastic models for haptic textures. In M. R. Stein, editor, Proc. SPIE Vol. 2901, p. 34-44, Telemanipulator and Telepresence Technologies III, pages 34-44, 1996.

[10] V. Hayward and O. R. Astley. Performance measures for haptic interfaces. In G. Giralt and G. Hirzinger, editors, Robotics Research: The 7th International Symposium, pages 195-207. Springer Verlag, 1996.
[11] V. Hayward, J. Choksi, G. Lanvin, and C. Ramstein. Design and multi-objective optimization of a linkage for a haptic interface. In J. Lenarcic and B. Ravani, editors, Advances in Robot Kinematics, pages 352359. Kluver Academic, 1994.

[12] V. Hayward, P. Gregorio, O. Astley, S. Greenish, M. Doyon, L. Lessard, J. McDougall, I. Sinclair, S. Boelen, X. Chen, J.-P. Demers, J. Poulin, I, Benguigui, N. Almey, B. Makuc, and X. Zhang. Freedom-7: A high fidelity seven axis haptic device with application to surgical training. In A. Casals and A. T. de Almeida, editors, Experimental Robotics V, pages 445-456. Springer Verlag, 1998. Lecture Notes in Control and Information Science 232.

[13] V. Hayward and D. Yi. Change of height: An approach to the haptic display of shape and texture without surface normal. In B. Siciliano and P. Dario, editors, Experimental Robotics VIII, pages 570-579. Springer Tracts in Advanced Robotics, Springer Verlag, 2003.

[14] F. Janabi-Sharifi, V. Hayward, and C.-S. J. Chen. Discrete-time adaptive windowing for velocity estimation. IEEE T. On Control Systems Technology, 8(6):1003-1009, 2000.

[15] T. H. Massie and J. K. Salisbury. The PHANToM haptic interface: A device for probing virtual objects. In Proc. ASME IMECE Symposium on Haptic Interfaces for Virtual Environments and Teleoperator Systems, DSC-Vol. 55-1, pages pp. 295-299, 1994.

[16] M. Minsky and S. J. Lederman. Simulated haptic textures: Roughness. In Proc. ASME IMECE Symposium on Haptic Interfaces for Virtual Environments and Teleoperator Systems, DSC-Vol. 58, pages 421426, 1996.

[17] M. A. Otaduy and M. C. Lin. A perceptually-inspired force model for haptic texture rendering. In Proc. 1st Symposium on Applied perception in graphics and visualization, pages 123-126. ACM Press, 2004.

[18] J. Siira and D.K. Pai. Haptic textures - a stochastic approach. In Proc. IEEE International Conference on Robotics \& Automation, pages 557-562, 1996.

[19] J. M. Weisenberger, M. J. Kreier, and M. A. Rinker. Judging the orientation of sinusoidal and squarewave virtual gratings presented via 2-DOF and 3DOF haptic interfaces. Haptics-e, 1(4), 2000. 